\title{
Thermal conductivity of Si nanostructures containing defects: Methodology, isotope effects, and phonon trapping
}

\author{
T. M. Gibbons, By. Kang, and S. K. Estreicher* \\ Physics Department, Texas Tech University, Lubbock, Texas 79409-1051, USA \\ C. Carbogno \\ Fritz-Haber-Institut der Max-Planck Gesellschaft, Faradayweg 4-6, D-14195 Berlin-Dahlem, Germany
}

(Received 27 April 2011; published 26 July 2011)

\begin{abstract}
A first-principles method to calculate the thermal conductivity in nanostructures that may contain defects or impurities is described in detail. The method mimics the so-called "laser-flash" technique to measure thermal conductivities. It starts with first-principles density-functional theory and involves the preparation of various regions of a supercell at slightly different temperatures. The temperature fluctuations are minimized without using a thermostat and, after averaging over random initial conditions, temperature changes as small as $5 \mathrm{~K}$ can be monitored (from 120 to $125 \mathrm{~K}$ ). The changes to the phonon density of states and the specific heat induced by several atomic percent of impurities are discussed. The thermal conductivity of Si supercells is calculated as a function of the temperature and of the impurity content. For most impurities, the drop in thermal conductivity is unremarkable. However, there exist narrow ranges of impurity parameters (mass, bond strength, etc.) for which substantial drops in the thermal conductivity are predicted. These drops are isotope dependent and appear to be related to the vibrational lifetime of specific impurity-related modes.
\end{abstract}

DOI: 10.1103/PhysRevB.84.035317

PACS number(s): 61.72.S-, 63.20.dk

\section{INTRODUCTION}

Semiconductors such as crystalline Si are easily doped with impurities. Some of them introduce charge carriers (electrons or holes) into the material, thus allowing the electrical conductivity to be controlled almost at will. Other types of impurities introduce deep levels in the forbidden energy gap of the material, become $e^{-}-h^{+}$recombination centers, and reduce charge-carrier lifetimes and concentrations. Other impurities yet increase the mechanical strength of the crystal, introduce optically active defect centers, or even allow the control of the spin of charge carriers. ${ }^{1-3}$

In a recent paper, ${ }^{4}$ we have initiated a study of the impact of impurities on the thermal properties of Si nanostructures. Being able to control the thermal conductivity $\kappa$ by doping could improve the heat dissipation on a chip, e.g., by implanting a "thermal circuit," analogous to an electrical circuit. It could also improve the thermoelectric figure of merit ${ }^{5}$ $Z T=S^{2} T \sigma / \kappa$ (where $S$ is the Seebeck coefficient and $\sigma$ the electrical conductivity) by allowing the control of the electrical and thermal conductivities independently.

We introduced ${ }^{4}$ a technique to calculate $\kappa$ from first principles in periodic supercells, which may contain defects. The electronic structure is obtained from standard density functional (DF) theory, which is commonly used to predict the properties of defects in semiconductors. ${ }^{6}$ Nonequilibrium $a b$ initio molecular dynamics (MD) simulations with minimal temperature fluctuations, without a thermostat, are made possible by carefully preparing the system slightly away from equilibrium. Supercell preparation is the key ingredient in the present calculations. The approach mimics the experimental "laser-flash" technique ${ }^{7}$ in which the temperature $T(x, t)$ is measured at a point $x$ as a function of time $t$ following a laser-pulse excitation at $x=0$ and $t=0$.

It is well known ${ }^{8,9}$ that the presence of any kind of defect in a material increases phonon scattering and therefore reduces $\kappa$.
Our earlier results ${ }^{4}$ confirm this qualitative statement. Indeed, the presence of a few atomic percent of impurities generally leads to a drop in $\kappa$ which, for most impurities, ranges from $\sim 20 \%$ to a factor of 2 relative to the pure material. However, theory also predicts that there exists a narrow range of defect parameters (isotopic mass, bond strength, etc.) for which a resonance occurs and $\kappa$ drops precipitously. As far as $\kappa$ is concerned, not all defects are created equal, just as is the case for $\sigma$.

Impurities in covalent crystals introduce unique, localized, vibrational modes. When the concentration of impurities is of the order of the atomic percent, these localized modes become vibrational bands which exhibit varying degrees of localization (see below). As heat propagates through the material from a warmer region to a colder one, these localized bands may absorb energy. At the atomic scale, the impact of impurities on the thermal conductivity depends on the lifetimes of these impurity-induced localized vibrational bands. In particular, modes with long lifetimes which trap and keep energy reduce the flow of heat and therefore $\kappa$.

It is necessary-but not sufficient-for a vibrational mode to exhibit a high degree of localization in order to have a long lifetime. Experimental ${ }^{10-12}$ and theoretical ${ }^{12-14}$ studies have shown that the lifetimes of highly localized vibrational modes of light impurities in Si can vary by up to two orders of magnitude. In some cases, a simple isotope substitution has a surprisingly large impact on the lifetime of a specific mode, as a fast two-phonon decay becomes a much slower three- or even four-phonon decay. ${ }^{12}$

Thus, no simple "rule of thumb" connects a specific type of impurity to high degrees of localization, long vibrational lifetimes, and/or a predictable impact on the thermal conductivity $\kappa$, which is a macroscopic quantity. The details of phonon coupling are far too complicated, and are often isotope dependent. Thus, experimental studies of the impact 
of impurities on the thermal conductivity of a covalent material such as Si would have to rely on an inefficient trial-and-error approach. First-principles theory should take the lead and provide some insights into this potentially important area of research.

Three theoretical approaches are commonly used to calculate the thermal conductivity of materials. The GreenKubo method involves equilibrium MD and is based on the fluctuation-dissipation theorem. The steady-state method is based on nonequilibrium MD with (usually very large) temperature gradients maintained between two parts of the system. Finally, the direct solution of the Boltzmann transport equation is also possible. For a long time, these approaches have been limited to semiempirical techniques which work well for defect-free materials. However, the transferability of any semiempirical method to systems containing impurities is questionable. ${ }^{6}$ First-principles methods are now more commonly used to calculate the force constants or other input parameters.

In the Green-Kubo method, ${ }^{15,16}$ the macroscopic fluxes of charge $(q)$ and heat $(h)$ are written in terms of the transport coefficients $L_{\alpha \beta}=L_{\beta \alpha}$ (where $\alpha, \beta$ stand for $q$ or $h$ ), which themselves are defined in terms of the correlation functions of the microscopic fluxes of charge and heat, $L_{\alpha \beta}=\frac{1}{3 \Omega} \int_{0}^{\infty}\left\langle\vec{J}_{\alpha}(t) \cdot \vec{J}_{\beta}(0)\right\rangle d t$, where $\Omega$ is the volume of the system, \langle\rangle is the average in thermal equilibrium, $\vec{J}_{q}=$ $\sum_{i} Z_{i} e \vec{v}_{i}$, and $\vec{J}_{h}=\frac{d}{d t} \sum_{i} E_{i} \vec{r}_{i}$, where $Z_{i} e, \vec{v}_{i}, E_{i}$, and $\vec{r}_{i}$ are the nuclear charge, velocity, energy, and position of atom $i$, respectively. Once the three $L_{\alpha \beta}$ coefficients are known, one obtains the electrical conductivity, thermopower, and thermal conductivity. The latter is $\kappa=\left(L_{q q} L_{h h}-L_{q h}^{2}\right) / L_{q q} k_{B} T^{2}$, but $L_{q h}$ is often small and assumed to be zero.

The problem is that the computation of the heat flux requires the knowledge of the total energies $E_{i}$ of each single atom, a quantity that is not directly accessible in first-principles calculations. An a posteriori decomposition of the total energy of a system into individual atomic contributions is error prone and cumbersome, if not impossible in the presence of defects. Hence the Green-Kubo approach has so far only been applied within the framework of semiempirical potentials, for instance, to calculate $\kappa$ in $\mathrm{Si}$ (Ref. 17) and its frequency dependence, ${ }^{18}$ in $\mathrm{Si} / \mathrm{Ge}$ superlattices, ${ }^{19,20}$ and in $\mathrm{Ge} .^{21}$

The steady-state method involves establishing a large temperature gradient and maintaining it with thermostats until the steady state is reached. Because of the large temperature fluctuations, very large temperature gradients are needed and the specific heat varies from one end of the system to the other. Very long MD runs are required to achieve a steady-state situation. The method has been used in conjunction with semiempirical potentials to calculate $\kappa$ in $\mathrm{C}$ nanotubes, ${ }^{22}$ in $\mathrm{Si}$ nanowires, ${ }^{23}$ in solid argon, ${ }^{24}$ and to study the effects of large vacancy clusters in $\mathrm{Si}^{25}$ The steady-state method has also been used to predict ${ }^{26}$ the high-temperature thermal conductivity of $\mathrm{MgO}$ using forces obtained from first-principles DF theory. The large temperature gradients required are better suited to very high temperatures than to the low temperatures considered here, where the specific heat varies considerably with $T$.

Finally, the Boltzmann transport equation can be solved directly to find $\kappa$. The equation contains the specific heat, the phonon group velocities, and the phonon lifetimes. The specific heat is readily obtained from first principles if the phonon density of states is known. The group velocities can be extracted from the phonon dispersion curves. But the vibrational lifetimes are more difficult to calculate. This has been done from first-principles autocorrelation functions in $\mathrm{MgO}$ (Ref. 27) and in elemental semiconductors with third-order force constants obtained from first-principles DF perturbation theory. ${ }^{28,29}$ The Boltzmann equation can also be solved using Green's functions to find mode-dependent transmission coefficients. This approach has been used to study the impact of impurities on the thermal conductivity of graphene. ${ }^{30}$ As mentioned above, the details of phonon coupling are more complicated in the case of defects, as the decays of localized modes involve the coupling of the defect-related modes with two to five (or more) phonons. ${ }^{12-14}$ As discussed below, when defects are present in high concentrations, localized modes can substantially reduce the thermal conductivity and the reduction is sometimes strongly isotope dependent.

Our nonequilibrium $a b$ initio MD approach starts with a supercell prepared slightly away from equilibrium in a manner that minimizes the thermal fluctuations and allows the use of very small temperature gradients. We monitor how the system returns to equilibrium without using a thermostat. We have tested the supercell preparation technique in conjunction with the calculations of the temperature and isotope dependence of vibrational lifetimes ${ }^{12-14}$ using the same first-principles approach. Our longest MD simulations, required for a very slowly decaying vibrational excitation, involved almost one million time steps. The amplitude of the temperature fluctuations at $200 \mathrm{~K}$ decreased very little throughout this MD run, demonstrating that, even at moderate temperatures, our supercell preparation technique does mimic thermal equilibrium with good accuracy.

In this paper, we extend our earlier work ${ }^{4}$ in several ways. In Sec. II, we describe our theoretical method in detail, including the specifics of supercell preparation, the normal-mode phase matching at the hot-cold interface, and the averaging required to obtain $\kappa$. In Sec. III, we discuss the changes introduced by impurities in the phonon density of states and the specific heat, predict the temperature dependence of the thermal conductivity in the $\mathrm{Si}_{192}$ supercell, the dependence of $\kappa$ on the concentration of impurities, and then focus on the change in $\kappa(T=125 \mathrm{~K})$ when the supercell contains 5.2 at.\% of vacancies or various isotopes of $\mathrm{C}, \mathrm{Si}, \mathrm{Fe}$, or $\mathrm{Ge}$. The strong variation of $\kappa$ with the impurity isotope is correlated to the localization of some impurity-related modes. The key results are discussed in Sec. IV.

\section{METHODOLOGY}

\section{A. Supercell construction}

The host crystal is represented by periodic supercells constructed by stacking slices along a specific crystalline direction. Most of our calculations are done with twelve 16-Si atoms slices stacked along the $\langle 100\rangle$ direction to create a parallelepiped which is $33 \AA$ long and has a diameter of 15 $\AA$. We have also used ${ }^{4}$ the $\mathrm{Si}_{384}$ and $\mathrm{Si}_{768}$ supercells (Fig. 1) to investigate the impact of the cross-sectional area on the 


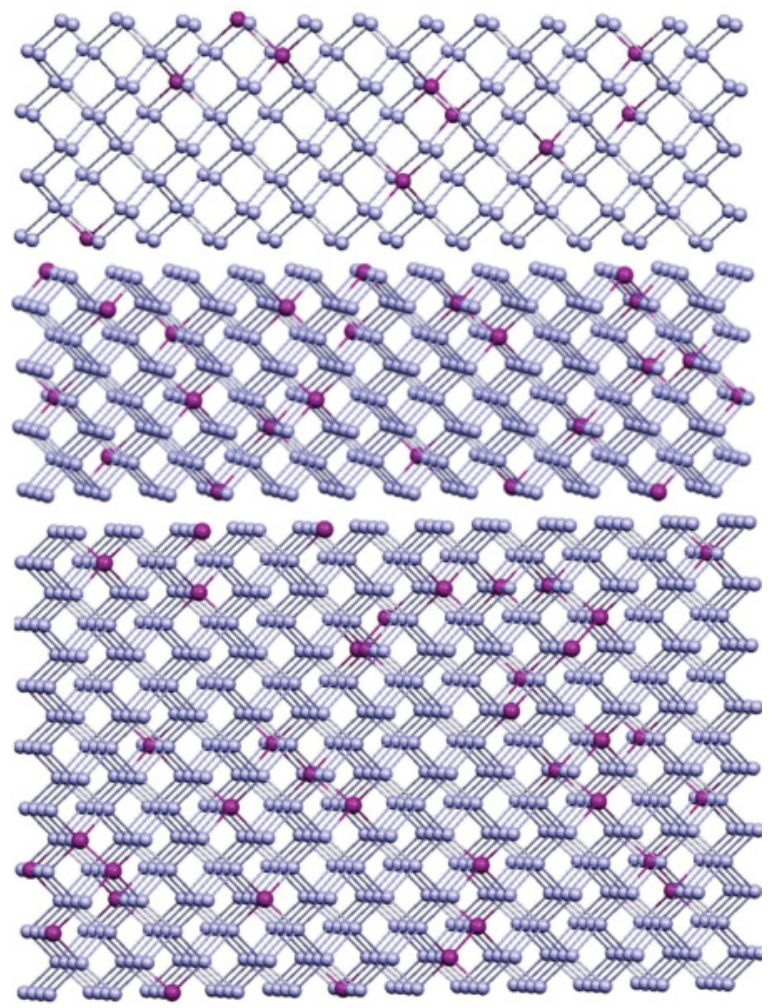

FIG. 1. (Color online) The $\mathrm{Si}_{192}, \mathrm{Si}_{384}$, and $\mathrm{Si}_{768}$ supercells consist of $12 \mathrm{Si}_{16}, \mathrm{Si}_{32}$, and $\mathrm{Si}_{64}$ slices, respectively, aligned along the $\langle 100\rangle$ direction. The supercells are shown with a random distribution of 5.2 at.\% of substitutional impurities [magenta (darker) atoms]. The three supercells are $3.3 \mathrm{~nm}$ long. Their diameters are 1.54, 2.44, and $4.50 \mathrm{~nm}$, respectively.

thermal conductivity. A similar construction can be performed for $\mathrm{Si}$ nanowires to investigate the impact of the surface conditions or impurity content on the thermal conductivity. ${ }^{31}$ Because the thermal conductivity calculations are highly computer intensive, we restrict the Brillouin-zone sampling to the $\Gamma$ point.

\section{B. Electronic structure method}

The first-principles DF calculations are carried out using the SIESTA package, ${ }^{32,33}$ but our method is not restricted to this particular electronic structure code. Indeed, it has also been successfully used to calculate the thermal conductivity of oxides $^{34}$ using the all-electron simulation package FHI-AIMS. ${ }^{35}$

In the SIESTA approach, the electronic core regions are removed from the calculations using ab initio norm-conserving pseudopotentials with the Troullier-Martins parametrization ${ }^{36}$ in the Kleinman-Bylander form. ${ }^{37}$ The SIESTA pseudopotentials have been optimized using the experimental bulk properties of the perfect solids and/or first-principles calculations $^{38}$ as well as vibrational properties of free molecules or known defects, when such experimental data are available.

The valence regions are treated using first-principles spin-DF theory with the exchange-correlation potential of Ceperley-Alder ${ }^{39}$ as parametrized by Perdew-Zunger. ${ }^{40}$ The calculations involving heavier elements such as $\mathrm{Fe}$ or $\mathrm{Ge}$ are treated within the generalized gradient approximation for the exchange-correlation potential. ${ }^{41}$ The basis sets for the valence states are linear combinations of numerical atomic orbitals. ${ }^{42,43}$ We use a double-zeta basis set for $\mathrm{H}, \mathrm{C}$, and $\mathrm{Si}$, and add polarizations functions for $\mathrm{Ge}$. The basis set for Fe consists of two sets of valence $s$ 's and $d$ 's and one set of $p$ 's. The charge density is projected on a real-space grid with an equivalent cutoff of 350 Ryd to calculate the exchange-correlation and Hartree potentials.

\section{Vibrational spectra}

The defect configurations must be optimized with care using a conjugate gradient algorithm. Our standard requirement is that the maximum force component be $0.003-0.001$ $\mathrm{eV} / \AA$. Supercell preparation relies on accurate dynamical matrices (see below), and unphysical negative frequencies appear when the geometries are insufficiently optimized. They indicate that the system is not at a true minimum of the $3 N$-dimensional potential energy surface ( $N$ is the number of atoms), i.e., that the curvature is negative in at least one direction.

The potential energy is sometimes very flat in one direction, and finding the precise minimum is computationally difficult. In such cases, we tolerate a few negative frequencies as long as their absolute value is small. A few of the orthonormal eigenvectors are incorrect, but the basis set is still complete and the error has a minor impact on supercell preparation.

Note that when dealing with a high concentration (a few atomic percent) of defects, conjugate gradient calculations often converge poorly. For example, the geometry optimizations with 5 at.\% of vacancies in the supercell properly converged only when we started by optimizing the supercell with a single vacancy, then again with two vacancies, etc., until all the vacancies were in place. Only then do we obtain dynamical matrices with no negative frequencies.

Once the geometry is properly optimized, the force constant matrix, and then the dynamical matrix are calculated. Its eigenvalues are all the normal-mode frequencies $\omega_{s}$ of the system. The eigenvectors $e_{\alpha i}^{s}(i=x, y, z)$ give the relative displacements of the nuclei $\alpha$ for each mode $s$. These $3 N$ orthonormal vectors are central to the present calculations as they allow us to prepare the supercell (or parts of the supercell) in thermal equilibrium at the temperature $T$. They also allow us to quantify the localization of any normal vibrational mode. Indeed, a quantitative measure of how localized a specific mode is on one atom or a group of atoms is provided by a plot of $L_{\{\alpha\}}^{2}=\left(e_{\alpha x}^{s}\right)^{2}+\left(e_{\alpha y}^{s}\right)^{2}+\left(e_{\alpha z}^{s}\right)^{2}$ vs $\omega_{s}$, where $\{\alpha\}$ is a single atom or the sum over a group of atoms.

\section{Supercell preparation in thermal equilibrium}

The central ingredient is the preparation of the supercell slightly away from thermal equilibrium at $t=0$. This is achieved by initializing the atoms in a linear combination of normal vibrational modes at the desired temperature. This involves random distributions of mode energies and phases.

In thermal equilibrium, the average energy of each mode is $k_{B} T$. We write $\left\langle E_{s}\right\rangle=\gamma_{s} k_{B} T$, where $\gamma_{s}=\int_{0}^{E_{s}}$ $\left\{e^{-E / k_{B} T} / k_{B} T\right\} d E$ defines a random distribution of mode 
energies that averages out to $k_{B} T$. This gives $\left\langle E_{s}\right\rangle=$ $-k_{B} T \ln \left(1-\gamma_{s}\right)$. Thus, the average kinetic energy of each mode is

$$
\left\langle K_{s}\right\rangle=-\frac{1}{2} k_{B} T \ln \left(1-\gamma_{s}\right), \quad 0<\gamma_{s}<1 .
$$

The Cartesian coordinates of atom $\alpha$ in the mode $s$ is related to the eigenvectors $e_{\alpha i}^{s}$ via the normal-mode coordinate $q_{s}(T)$, which we assume to be harmonic

$$
r_{\alpha i}^{s}=q_{s}(T) e_{\alpha i}^{s}=\frac{1}{\sqrt{m_{\alpha}}} A_{s}(T) \cos \left(\omega_{s} t+\varphi_{s}\right) e_{\alpha i}^{s} .
$$

The relative phase $\varphi_{s}$ of each mode is in the range $[0,2 \pi[$.

The harmonic assumption for the initial state of the supercell is never perfect since even the zero-point motion is slightly anharmonic. ${ }^{44}$ The assumption becomes increasingly worse as the temperature increases. However, it is only used for supercell preparation at $t=0$. It is simple, produces remarkably constant temperature fluctuations, and obviates the need for a thermostat.

If $\left(\vec{r}_{\alpha}^{s}\right)^{2}=\sum_{i}\left\{r_{\alpha i}^{s}\right\}^{2}$, the average kinetic energy of the mode $s$ is

$$
\begin{aligned}
\left\langle K_{s}\right\rangle & =\frac{1}{2} \sum_{\alpha} m_{\alpha}\left\langle\left(\dot{\vec{r}}_{\alpha}^{s}\right)^{2}\right\rangle \\
& =\frac{1}{2} \omega_{s}^{2} A_{s}^{2}(T) \frac{1}{2} \sum_{\alpha i}\left\{e_{\alpha i}^{s}\right\}^{2}=\frac{1}{4} \omega_{s}^{2} A_{s}^{2}(T) \\
& =-\frac{1}{2} k_{B} T \ln \left(1-\gamma_{s}\right) .
\end{aligned}
$$

Thus, in thermal equilibrium at the temperature $T$, the mode amplitudes are given by

$$
A_{s}(T)=\frac{\sqrt{-2 k_{B} T \ln \left(1-\gamma_{s}\right)}}{\omega_{s}},
$$

which allows the calculation of the initial positions and velocities of all the atoms in the supercell in (very near) thermal equilibrium. The price we pay for eliminating the need for a thermalization and a thermostat is that we introduce two random quantities: the initial phases and initial energies of the normal vibrational modes. The results must be averaged over a statistically relevant number of initial conditions (see below). However, this averaging further reduces the magnitude of the temperature fluctuations.

\section{E. Preparation away from equilibrium and the hot-cold interface}

When calculating vibrational lifetimes, ${ }^{12-14}$ the supercell is prepared away from equilibrium by exciting one specific vibrational mode by one phonon, that is, using the appropriate eigenvector of the dynamical matrix to assign to this mode the initial potential energy $3 \hbar \omega / 2$ (zero-point energy plus one phonon). This guarantees that the classical oscillator starts with the same oscillation amplitude (same amount of anharmonicity) as the quantum oscillator in the sample being measured.

In the present work, we prepare one slice of the supercell at a higher temperature than the rest. For example, consider a supercell divided into $N=12$ slices. If we prepare the first slice at $T_{\text {hot }}=180 \mathrm{~K}$ and the other 11 at $T_{\text {cold }}=120 \mathrm{~K}$, the final (equilibrium) temperature will be $125 \mathrm{~K}$. The temperature in the central slice, where $T(t)$ is calculated, increases by only $5 \mathrm{~K}$. Even smaller temperature changes can be monitored at lower temperatures. We have tested this with averages including up to 120 runs. This number can be reduced if the modes at the hot-cold interface are prepared in phase at $t=0$. Further, it is important that the initial temperature of the central slice-not just the initial average temperature of the 11 cold slices - be at the desired temperature. Thus, in each run, we want (a) the temperature of the first slice to be $T_{\text {hot }}$, (b) the average temperature of the other 11 slices to be $T_{\text {cold }}$, and (c) the temperature of the central slice to be as close as possible to $T_{\text {cold }}$ as well. This is achieved in the following way.

First, we randomly generate initial mode phases and energies (of the entire supercell) consistent with $T_{\text {cold }}$ until the temperature of the central slice and the average temperature of the 11 cold slices are within $0.5 \%$ of $T_{\text {cold }}$. From now on, the phases are kept constant. Second, we scale the mode amplitudes (of the entire supercell) until the first slice is exactly at $T_{\text {hot }}$. Third, we apply the $T_{\text {hot }}$ conditions to all the atoms in the first slice and the $T_{\text {cold }}$ conditions to the atoms in the 11 other slices. Thus, requirements (a), (b), and (c) listed above for supercell preparation away from equilibrium are satisfied to a high degree of accuracy, and the modes are in phase at the hot-cold interface.

\section{F. Averaging and thermal conductivity}

MD runs without a thermostat in the prepared supercell allow the calculation of $T(t)$ in the central slice. The time step is selected to be $1 / 40$ th-1/30th of the shortest oscillation period in the system. Because of the periodic boundary conditions applied, half of the temperature increase in the central slice comes from the hot slice and the other half from its nearest image outside the supercell. Thus, the temperature in the central slice increases because of a heat flux from both sides, and depends on the impurity distribution in the entire supercell.

The preparation procedure is repeated as many times as necessary until the fit (see below) to the calculated $T(t)$ produces a converged $\kappa\left(T_{\text {final }}\right)$. Because of the random nature of the initial normal-mode phases and energies, the temperature versus time behavior must be monitored in each run for the occasional bizarre behavior resulting from a rare coincidence of phase and/or energy between strongly coupled modes. For example, the temperature of the central may suddenly drop instead of increasing. Such runs are discarded. This would be irrelevant when averaging over a a huge number of runs and/or when using very large supercells, but should be monitored when only a few dozen runs are computationally practical and relatively small supercells are used. If the supercell is carefully prepared, 60 runs normally suffice for a properly converged value (Fig. 2). However, when dealing with a heavily perturbed system-such as 5.2 at.\% of vacancies or impurities which produce a large reduction in thermal conductivity - additional averaging is sometimes necessary.

The averaged temperature $T(t)$ of the central slice is fit to the analytic solution to the heat diffusion equation, which is the same function used by experimentalists. ${ }^{7}$ Adapted to the 


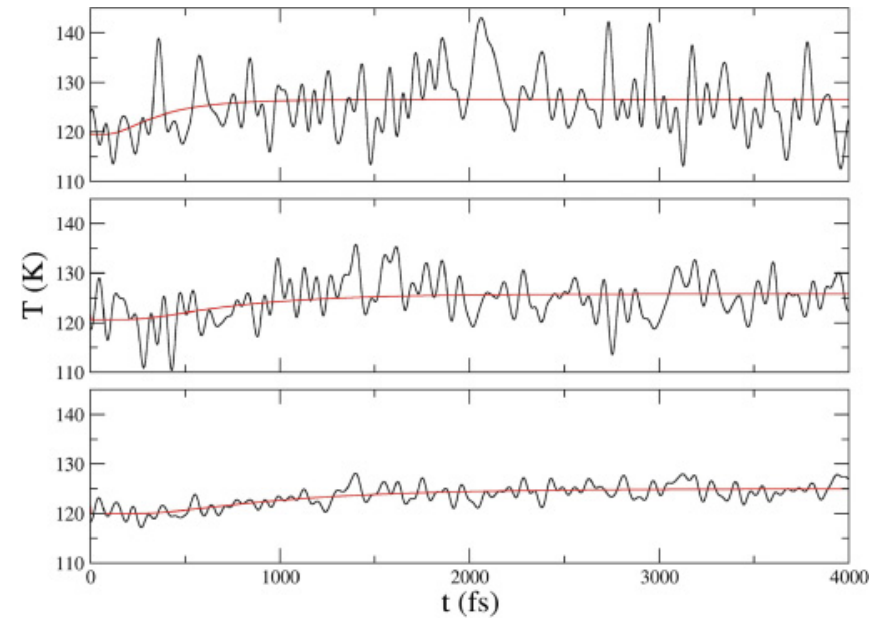

FIG. 2. (Color online) Effect of averaging for $T(t)$ in the central slice in the ${ }^{28} \mathrm{Si}_{162}{ }^{56} \mathrm{Si}_{30}$ supercell after 1 run (top) and after averaging over 30 (middle) and 60 (bottom) runs. With $T_{\text {hot }}=180 \mathrm{~K}$ and $T_{\text {cold }}=$ $120 \mathrm{~K}$, the temperature in the central slice increases by $5 \mathrm{~K}$. Note that the temperature fluctuations drop to just a few degrees $\mathrm{K}$ after averaging. The (red) solid line shows the fit from which we extract $\kappa$.

present configuration, this is

$T(x, t)=T_{\text {cold }}+\left(T_{\text {final }}-T_{\text {cold }}\right) \sum_{n}(-1)^{n} \exp \left\{-n^{2} \pi^{2} \alpha t / x^{2}\right\}$

and $\kappa=\alpha \rho C$, where $\rho$ is the density of the material and $C$ the calculated specific heat at the appropriate temperature. For example, if the temperature of the central slice increases from 120 to $125 \mathrm{~K}$, we use the calculated $C_{v}$ at the average $122.5 \mathrm{~K}$. Accurate specific heats can easily be calculated once the dynamical matrix is known. ${ }^{45-49}$ Our sum runs from $n=0$ to $n=10$.

\section{G. Validity of the fitting equation}

The fitting equation we use to extract the thermal conductivity was developed for macroscopic samples. It is not obvious that it can be used in our nanostructures as well. This equation assumes that Fourier's law is valid. It also contains the specific heat, which varies with temperature. Finally, it assumes that we are in the diffusion, not the ballistic, regime.

All the results we obtained to date (see Ref. 4 and the results discussed in this paper) suggest that our predictions are sound. For example, the calculated $\kappa(T=125 \mathrm{~K})=$ $2.1 \times 10^{-2} \mathrm{~W} / \mathrm{cm} \mathrm{K}$ is larger than, but consistent with, that of Si nanowires of small diameter. ${ }^{50-53}$ This discrepancy is expected since phonon scattering at the surface reduces the thermal conductivity of nanowires (especially if the surface is heavily damaged ${ }^{54}$ ), while we use periodic boundary conditions and have no surface. Further, the cross-sectional area of our supercell is smaller than that of the smallest nanowire measured to date. The $\kappa(T=125 \mathrm{~K})$ of a $22-\mathrm{nm}$ diameter (our 1.5-nm supercell) is measured (calculated) to be 150 (300) times smaller than the bulk,,$^{55} 6.0 \mathrm{~W} / \mathrm{cm} \mathrm{K}$.

Our calculated $\kappa$ increases with supercell diameter. In the $\mathrm{Si}_{192}, \mathrm{Si}_{512}$, and $\mathrm{Si}_{768}$ supercells, we obtain $\kappa(125 \mathrm{~K})=2.1 \times$ $10^{-2}, 2.6 \times 10^{-2}$, and $4.5 \times 10^{-2} \mathrm{~W} / \mathrm{cm} \mathrm{K}$, respectively. The diameters of these supercells are $1.54,2.44$, and $4.50 \mathrm{~nm}$,

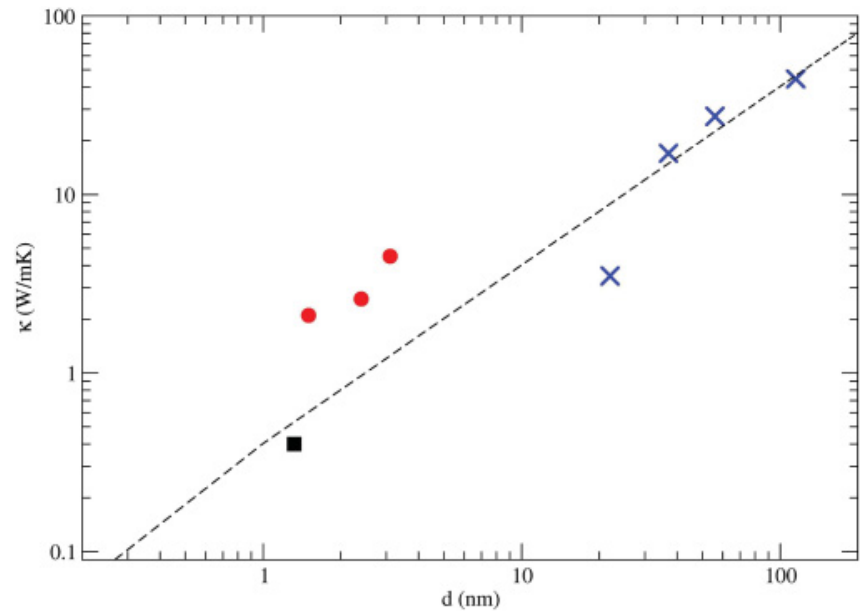

FIG. 3. (Color online) Thermal conductivities at $T=125 \mathrm{~K}$ measured in Si nanowires of various diameters [blue crosses- the values are extracted from Fig. 3(a) in Refs. 50 and 51] vs calculated values in the $\mathrm{Si}_{192}, \mathrm{Si}_{512}$, and $\mathrm{Si}_{768}$ supercells (red circles-these supercells have no surface) and in the $\mathrm{H}$-saturated $\mathrm{Si}_{200} \mathrm{H}_{32}$ nanowire (Ref. 31) (solid square). The thin dashed line is a linear fit to the four experimental points, where we assume that a zero-diameter nanowire has zero thermal conductivity.

respectively. Figure 3 shows that the calculated $\kappa(T=125 \mathrm{~K})$ in the H-terminated $\mathrm{Si}_{200} \mathrm{H}_{32}$ nanowire ${ }^{31}$ is consistent with the value extrapolated from existing measurements..$^{50,51}$

Our method is not applicable to arbitrary small supercells. The fitting function used to determine the thermal conductivity is only valid in the diffusive regime of heat transfer, i.e., when the length of the supercell is larger than the mean free path of the heat carrier. ${ }^{56}$ If the simulated supercell is too small, ballistic effects dominate the heat transfer and hence the simulated temperature profile exhibits a spatial and temporal behavior that is not compatible with our fitting function. ${ }^{57}$ In our case, the temperature changes we monitor are only a few degrees $\mathrm{K}$, the simulations closely follow the heat diffusion equation (Fig. 2) and ballistic effects are expected to be very small.

\section{H. Quantum corrections to $T$ and $\kappa$}

In classical MD simulations, the temperature $T_{\mathrm{MD}}$ is obtained from the kinetic energy of the nuclei

$$
E=2 \sum_{\alpha} \frac{1}{2} m_{\alpha} v_{\alpha}^{2}=3 N k_{B} T_{\mathrm{MD}} .
$$

As discussed by Volz et al. ${ }^{19,20}$ the quantum temperature $T$ is obtained from

$$
E=\int_{0}^{\infty} g(\omega) n(\omega, T) \hbar \omega d \omega,
$$

where $g(\omega)$ is the phonon density of states and $n(\omega, T)$ the Bose-Einstein distribution function. Note that the zero-point energy is ignored here so that the two temperature scales have the same zero.

In order to get the correction to the thermal conductivity, Volz et al. assume Fourier's law and write that, for a fixed 




FIG. 4. Quantum correction factor $d T / d T_{\mathrm{MD}}$ to the thermal conductivity $\kappa$ plotted as a function of $T$.

temperature gradient $\Delta T=\Delta T_{\mathrm{MD}}$, the ratio of the fluxes of heat $j$ to the thermal conductivity $\kappa$ are equal

$$
\frac{1}{\kappa_{\mathrm{MD}}} \frac{d j}{d T_{\mathrm{MD}}}=\frac{1}{\kappa} \frac{d j}{d T}=\frac{1}{\kappa} \frac{d j}{d T_{\mathrm{MD}}} \frac{d T_{\mathrm{MD}}}{d T}
$$

and therefore

$$
\kappa=\kappa_{\mathrm{MD}} \frac{d T_{\mathrm{MD}}}{d T} .
$$

Figure 4 shows the quantum correction factor $d T / d T_{\mathrm{MD}}$ to the thermal conductivity $\kappa$ vs $T$. It is applied in our calculations.

\section{RESULTS}

\section{A. Impact of impurities on the phonon density of states and the specific heat}

When the crystal contains small concentrations of impurities, new localized modes appear in an otherwise little-changed phonon density of state. However, when a few atomic percent of a substitutional impurity $X$ is present, the total number of normal modes remains constant but the presence of a large number of $\mathrm{Si}-X$ (instead of $\mathrm{Si}-\mathrm{Si}$ ) stretch modes strongly affects the optical region of $g(\omega)$. This is illustrated in Fig. 5, which compares the $g(\omega)$ calculated from the perfect ${ }^{28} \mathrm{Si}_{192}$ supercell to those from the ${ }^{28} \mathrm{Si}_{182}{ }^{12} \mathrm{C}_{10},{ }^{28} \mathrm{Si}_{182}{ }^{56} \mathrm{Fe}_{10}$, and ${ }^{28} \mathrm{Si}_{182}{ }^{74} \mathrm{Ge}_{10}$ supercells.

The consequence of these impurity-related changes is visible in the specific heat. In the harmonic approximation and at constant volume, ${ }^{46,49}$ it is given by

$$
C_{v}(T)=\frac{d}{d T} \int \hbar \omega g(\omega) n\left(\hbar \omega / k_{B} T\right) d \omega .
$$

The integral depends on the entire phonon density of states. One would expect that if the flow of heat decreases, then more thermal energy remains in the material and the specific heat increases. This increase is shown in Fig. 6 for ${ }^{28} \mathrm{Si}_{192}$, ${ }^{28} \mathrm{Si}_{182}{ }^{12} \mathrm{C}_{10},{ }^{28} \mathrm{Si}_{182}{ }^{56} \mathrm{Fe}_{10}$, and ${ }^{28} \mathrm{Si}_{182}{ }^{74} \mathrm{Ge}_{10}$. However, the magnitude of the change $\Delta C_{v}$ is not quantitatively related to the changes in $\kappa$ (see below).
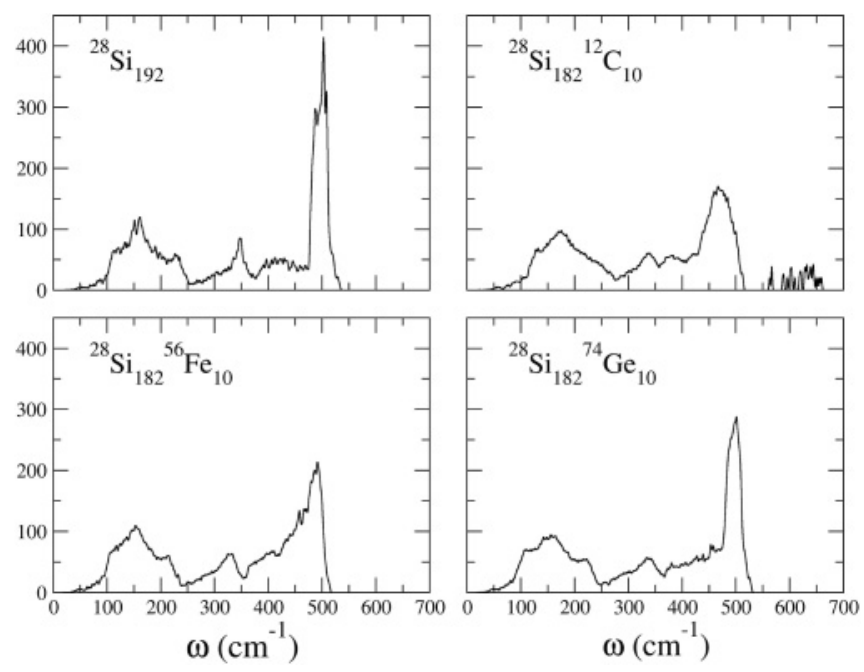

FIG. 5. Phonon density of states $g(\omega)$ obtained with a $50 q$-point sampling (and smoothing with narrow Gaussians) with ${ }^{28} \mathrm{Si}_{192}$ (top left-hand side), ${ }^{28} \mathrm{Si}_{182}{ }^{12} \mathrm{C}_{10}$ (top right-hand side), ${ }^{28} \mathrm{Si}_{182}{ }^{56} \mathrm{Fe}_{10}$ (bottom left-hand side), and ${ }^{28} \mathrm{Si}_{182}{ }^{74} \mathrm{Ge}_{10}$ (bottom right-hand side). Note that the most dramatic changes occur in the optical region, between 400 and $\sim 500 \mathrm{~cm}^{-1}$.

\section{B. Temperature dependence $\kappa(\mathrm{T})$}

In bulk semiconductors and insulators, the thermal conductivity increases as $T^{3}$ starting from $T=0 \mathrm{~K}$ until it reaches its peak value at a characteristically low temperature ${ }^{58-60} T_{\max }$. Since $\kappa=\alpha \rho C$, the specific heat also increases as $T^{3}$ in this temperature range. The increase is due to the occupation of higher-energy vibrational modes with $T$. However, the number of umklapp processes also increases with temperature, and these resistive processes begin to dominate at $T_{\max }$, beyond which the thermal conductivity decreases with $T{ }^{8,9} \mathrm{In} \mathrm{Si}$ nanowires ${ }^{50} T_{\max }$ is much higher and the magnitude of $\kappa\left(T_{\max }\right)$ considerably smaller than in bulk $\mathrm{Si}^{58}$ The same holds in our supercells. This is illustrated in Fig. 7, which shows $\kappa(T)$ calculated in ${ }^{28} \mathrm{Si}_{192}$ and ${ }^{28} \mathrm{Si}_{182}{ }^{56} \mathrm{Si}_{10}$. Note that the "impurity"

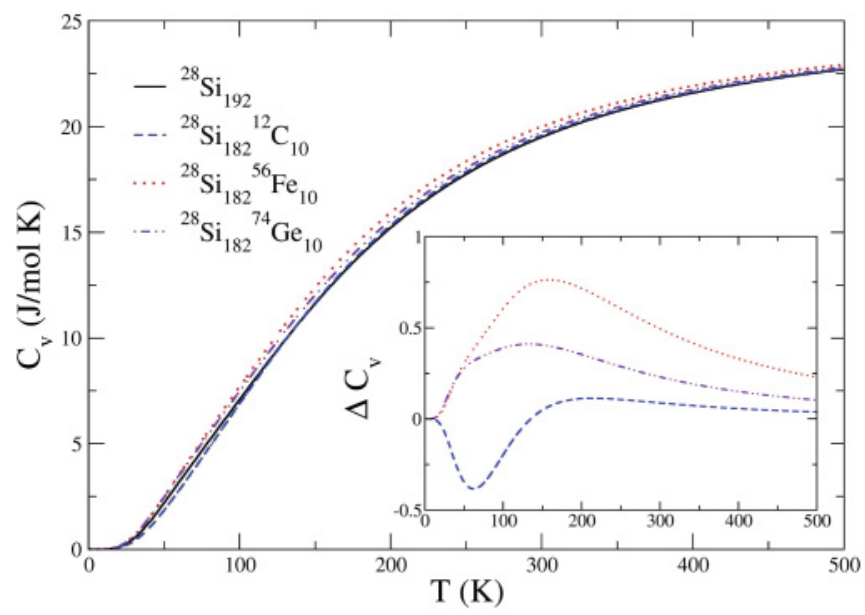

FIG. 6. (Color online) Specific heat of the ${ }^{28} \mathrm{Si}_{192}$ (solid black line), ${ }^{28} \mathrm{Si}_{182}{ }^{12} \mathrm{C}_{10}$ (dashed blue line), ${ }^{28} \mathrm{Si}_{182}{ }^{56} \mathrm{Fe}_{10}$ (dotted red line), and ${ }^{28} \mathrm{Si}_{182}{ }^{74} \mathrm{Ge}_{10}$ (dotted-dashed purple line). The differences relative to $\mathrm{C}_{v}\left({ }^{28} \mathrm{Si}_{192}\right)$ are shown in the inset. 




FIG. 7. (Color online) Log-log plot of $\kappa$ vs $T$ calculated in ${ }^{28} \mathrm{Si}_{192}$ and ${ }^{28} \mathrm{Si}_{182}{ }^{56} \mathrm{Si}_{10}$. The temperature $T_{\max }$ at which $\kappa$ is maximum and the low value of $\kappa\left(T_{\max }\right)$ are consistent with the measurements in $\mathrm{Si}$ nanowires (Refs. 50 and 51) (see Fig. 3). Note that, except for the scaling, the isotope effect is very similar to that observed for bulk Si (Ref. 58).

we use here is the nonexistent isotope 56 of $\mathrm{Si}$, as this value of the mass results in a large change in $\kappa$ (see below).

In the $\mathrm{Si}_{192}$ supercell, $\kappa$ reaches its maximum value at $227 \mathrm{~K}$, and $\kappa(227)=10.7 \mathrm{~W} / \mathrm{mK}$. At that temperature, the thermal conductivity for the $\mathrm{Si}_{182}{ }^{56} \mathrm{Si}_{10}$ isotope combination drops by $\sim 80 \%$, to $2.0 \mathrm{~W} / \mathrm{mK}$.

\section{Concentration dependence}

We also calculated the dependence of $\kappa(T=125 \mathrm{~K})$ on the concentration of impurities. Figure 8 shows $\kappa$ as a function of the concentration of ${ }^{56} \mathrm{Si}$ in the $\mathrm{Si}_{192}$ supercell. The behavior we find is comparable to that predicted for vacancy clusters in $\mathrm{Si}$ (Ref. 25) and for ${ }^{14} \mathrm{C}$ isotopes in ${ }^{12} \mathrm{C}$ graphene, ${ }^{61}$ as well as that predicted and measured in Y-stabilized zirconia. ${ }^{62}$

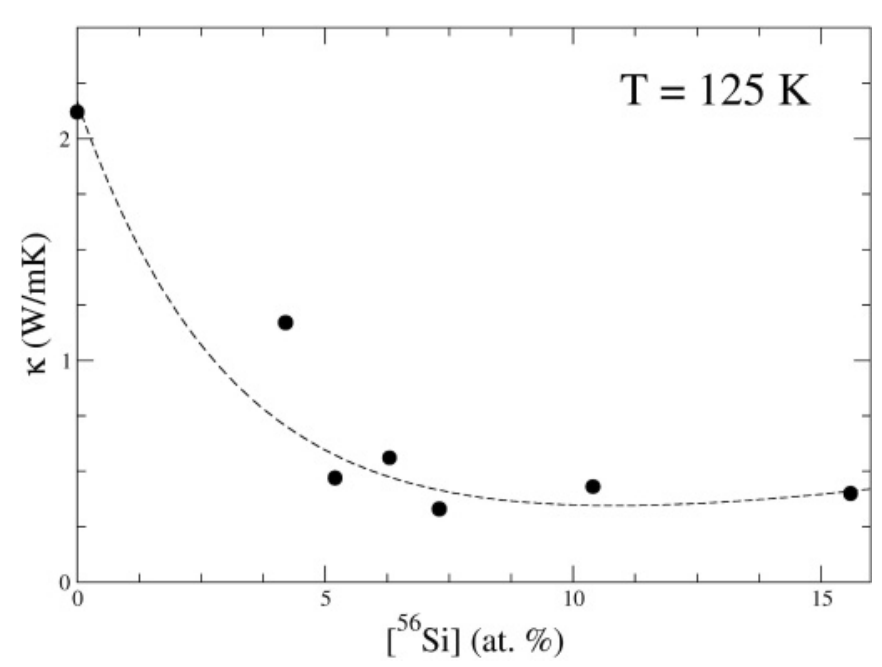

FIG. 8. Dependence of $\kappa$ on the concentration of ${ }^{56} \mathrm{Si}$ in the $\mathrm{Si}_{192}$ supercell.

\section{Dependence of $\boldsymbol{\kappa}$ on the mass and type of impurity}

If the material contains impurities, a reduction in the value of $\kappa$ relative to the impurity-free material is always observed. This is due to the scattering of phonons at impurities. What is unexpected is that the reduction sometimes varies in a strongly nonlinear fashion with the isotope of the impurity.

Our first study ${ }^{4}$ of the impurity dependence of $\kappa$ at a fixed temperature involved 5.2 at.\% of impurities. This large concentration of impurities was selected because the large changes in thermal conductivity measured ${ }^{58}$ as a function of the isotopic purity of $\mathrm{Si}$ involved several atomic percent of ${ }^{29} \mathrm{Si}$ and ${ }^{30} \mathrm{Si}$. However, the "impurities" used in Ref. 4 were simply (invented) $\mathrm{Si}$ isotopes with arbitrarily small or large mass, ${ }^{M} \mathrm{Si}$. This approach was based on the following argument. Impurities introduce unique localized vibrational modes into the phonon density of states, and we wanted to see how $\kappa$ varies when the frequencies of these modes change. These frequencies depend mostly on the impurity mass and on the Si-impurity bond strength. The simplest way to vary the frequencies of the impurity-related bands is to vary the mass. This greatly facilitates the calculations since no additional geometry optimizations are required and the same force constant matrix can be used. $\kappa(T=125 \mathrm{~K}, M)$ calculated in ${ }^{28} \mathrm{Si}_{182}{ }^{M} \mathrm{Si}_{10}$ exhibits a sharp drop for $M=56$. However, this approach has little impact on the phonon density of states since the bond strengths remain constant.

We have now extended this study to ${ }^{28} \mathrm{Si}_{182}{ }^{M} X_{10}$ for $X=\mathrm{C}$, $\mathrm{Fe}$, and Ge. We also considered the case $M=0$, that is, 5.2 at. $\%$ of $\mathrm{Si}$ vacancies. The results are shown in Fig. 9. The dependence of the thermal conductivity on the impurity isotope is highly unexpected.

A concentration of $5.2 \%$ of vacancies in the material causes $\kappa$ to drop by a factor of $\sim 2.5$. A substantial drop in the thermal conductivity has indeed been mentioned in conjunction with a $\mathrm{Si}$ surface exposed to a hydrogen plasma, which is known to generate damage near the surface of $\mathrm{Si}^{63}$ These studies

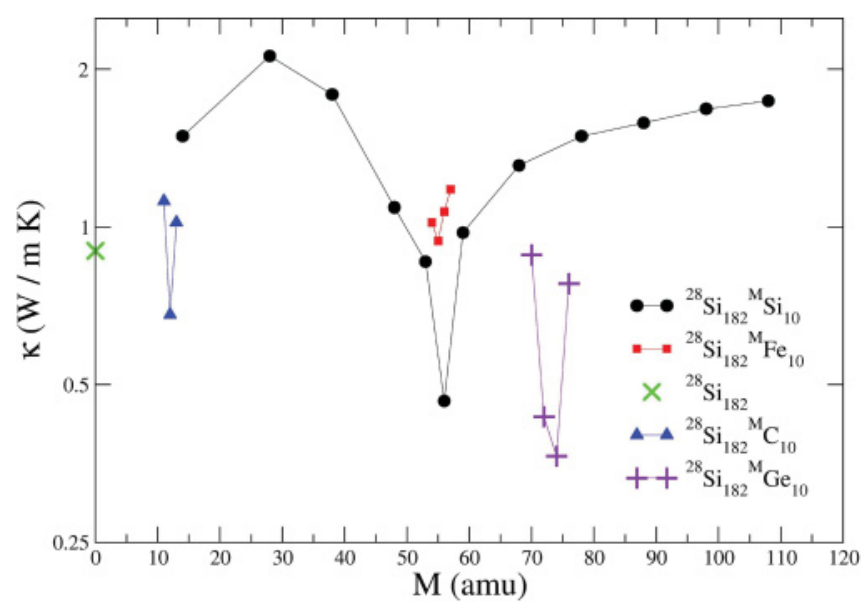

FIG. 9. (Color online) $\kappa(T=125 \mathrm{~K})$ in the 192-atom supercell containing 5.2 at.\% impurities. The (black) dots are for ${ }^{28} \mathrm{Si}_{182}{ }^{M} \mathrm{Si}_{10}$ (note: most of these $\mathrm{Si}$ "isotopes" do not exist); the (green) $\times$ for ten vacancies in the supercell; the (blue) triangles for ${ }^{28} \mathrm{Si}_{182}{ }^{M} \mathrm{C}_{10}$ (note: the ${ }^{11} \mathrm{C}$ does not exist); the (red) squares for ${ }^{28} \mathrm{Si}_{182}{ }^{M} \mathrm{Fe}_{10}$ with $M=54,55,56$, and 57; the (purple) crosses for ${ }^{28} \mathrm{Si}_{182}{ }^{M} \mathrm{Ge}_{10}$ with $M=70,72,74$, and 76 . 
focused on the behavior of ortho- and para- $\mathrm{H}_{2}$ molecules in the subsurface region of $\mathrm{Si}$, and neither the damage on this $\mathrm{Si}$ surface nor the change in thermal conductivity have been quantified. The experimental observation is nonetheless consistent with our prediction.

$\kappa$ is strongly affected by the isotope of all the impurities we have studied ( $\mathrm{C}, \mathrm{Si}, \mathrm{Fe}$, and $\mathrm{Ge})$. As mentioned above, if the impurity is ${ }^{M} \mathrm{Si}, \kappa$ sharply drops for the nonexistent ${ }^{56} \mathrm{Si}$ isotope. A qualitatively identical behavior is predicted when ${ }^{28} \mathrm{Si}$ is doped with 5.2 at.\% of $\mathrm{C}, \mathrm{Fe}$, and Ge. The drop occurs for ${ }^{12} \mathrm{C}$ (we invented ${ }^{11} \mathrm{C}$ and it shows $\kappa$ bouncing back upward), ${ }^{55} \mathrm{Fe}$, and ${ }^{74} \mathrm{Ge}$. The magnitude of the drop depends on the impurity, and some isotopes reduce $\kappa$ much more than others.

Thus, the qualitative statement that impurities lower the thermal conductivity because of phonon scattering is true but imprecise. That behavior is expected if one uses a mix of isotopes. However, we predict here that 5.2 at.\% of ${ }^{74} \mathrm{Ge}$ introduced into isotopically pure ${ }^{28} \mathrm{Si}$ has a much greater impact on the thermal conductivity than the same amount of ${ }^{70} \mathrm{Ge}$. This prediction can be tested experimentally.

Unexpectedly large isotope effects have been reported when measuring the vibrational lifetimes of impurity-related vibrational modes. Such effects can be dramatic when substituting $\mathrm{D}$ for $\mathrm{H},{ }^{11}$ but one could argue that the mass changes by a factor of 2. However, the lifetime of the asymmetric stretch of interstitial $\mathrm{O}$ in $\mathrm{Si}$ is highly sensitive to the isotope of one of its $\mathrm{Si}$ neighbors. Indeed, substituting ${ }^{28} \mathrm{Si}_{-}{ }^{16} \mathrm{O}-{ }^{28} \mathrm{Si}$ by ${ }^{29} \mathrm{Si}_{-}{ }^{16} \mathrm{O}-{ }^{28} \mathrm{Si}$ shifts the asymmetric stretch frequency by $0.1 \%$ but the vibrational lifetime increases by over $70 \% .^{12}$

\section{E. Correlation between the isotope dependence of $\kappa$ and the localization of impurity-related modes}

The very large isotope effects on the calculated thermal conductivity in Si nanostructures containing impurities and on the measured and calculated vibrational lifetimes of impurityrelated local vibrational modes lead us to suspect that the two phenomena are connected. Indeed, should a localized vibrational mode trap energy, it will remain in the excited state until the excitation decays into some linear combination of lower-frequency phonons. The longer the lifetime, the longer the energy remains trapped instead of propagating, and the lower the thermal conductivity. This process can be called "phonon trapping." 64

Vibrational lifetimes can be calculated ${ }^{12-14}$ using our supercell preparation technique, but the calculations are computer intensive even when we know precisely which mode is excited. In the present case, we deal with dozens of modes which exhibit various degrees of localization. The normal-mode frequencies and eigenvectors are calculated at $T=0 \mathrm{~K}$, a temperature at which the thermal conductivity is zero. At finite temperatures, the frequencies of different modes shift by different amounts. Calculating the lifetimes of all the possible modes for all the possible isotopes becomes an enormous computational task, especially if the lifetime of some modes becomes very long. A much simpler calculation involves the localization $L_{\{\alpha\}}^{2}=\left(e_{\alpha x}^{s}\right)^{2}+\left(e_{\alpha y}^{s}\right)^{2}+\left(e_{\alpha z}^{s}\right)^{2}$ of selected modes as a function of the isotopic mass of the impurity.

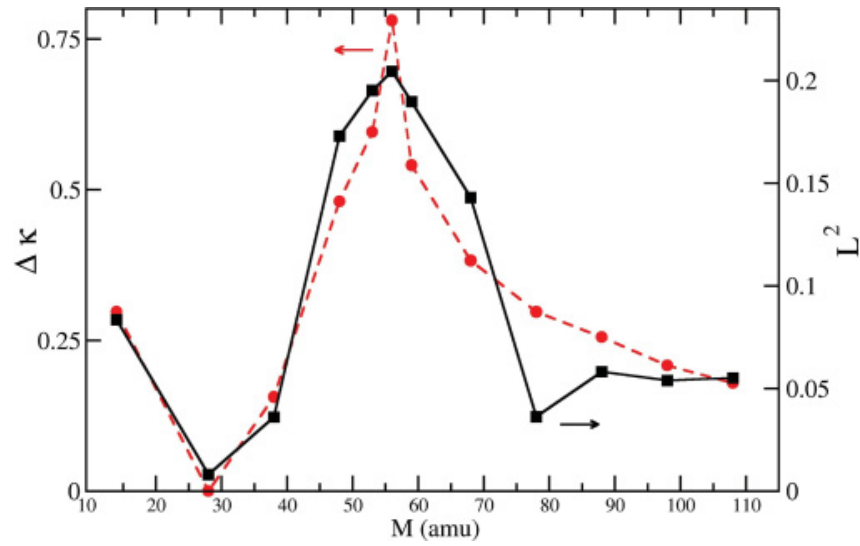

FIG. 10. (Color online) Correlation between the localization $L^{2}=L^{2}\left(\operatorname{Si}_{\mathrm{NN}}\right)\left(0<L^{2}<1\right.$ by definition $)$ of the breathing mode of the four NN to the impurity (black line, right-hand scale) and the change of thermal conductivity $\Delta \kappa=\left\{\kappa\left({ }^{28} \mathrm{Si}_{192}\right)-\right.$ $\left.\kappa\left({ }^{28} \mathrm{Si}_{182}{ }^{M} \mathrm{Si}_{10}\right)\right\} / \kappa\left({ }^{28} \mathrm{Si}_{192}\right)$ at $125 \mathrm{~K}$ (red dashed line, left-hand scale).

We started this analysis by looking at the modes that involve a substantial amount of impurity motion. However, the most promising mode proved to be the breathing oscillation of the four Si nearest neighbors (NNs) of the impurity, which involves no impurity motion. Note that four $\mathrm{Si}$ atoms participate in this mode for each impurity present. Thus, the 5.2 at.\% concentration implies that over $20 \%$ of the host atoms in the crystal could be involved in phonon trapping. Figure 10 shows the correlation between the localization $L^{2}$ of the breathing mode of the four NN to the impurity and the percent change of the thermal conductivity relative to the isotopically pure ${ }^{28} \mathrm{Si}_{192}$ supercell.

As discussed above, localization by itself is not a proof of long vibrational lifetime. Some highly localized modes have very short lifetimes. However, all the modes that exhibit a long lifetime are strongly localized. Thus, the striking correlation between $L^{2}$ and the change $\Delta \kappa$ as a function of the isotopic mass is not a proof but a strong hint that the underlying cause for the isotope effect on the thermal conductivity is related to the vibrational lifetimes of localized modes associated with the impurity.

\section{KEY POINTS AND DISCUSSION}

A new method for calculating entirely from first principles the thermal conductivity in nanostructures containing defects has been discussed in detail. The method minimizes the thermal fluctuations and allows us to monitor changes of temperature as small as $5 \mathrm{~K}$, from $T=120$ to $125 \mathrm{~K}$, without using a thermostat. The results must be averaged over two initial conditions: the relative phases and energies of all the modes at the time $t=0$. However, MD simulations can be performed without thermalization or thermostat. This method is used here to prepare the supercell slightly away from equilibrium, and then monitor how it returns to equilibrium. The calculation of thermal conductivities mimics the experimental laser-flash method.

The impact of the presence of a few atomic percent of impurities on the phonon density of states shows that all of $g(\omega)$ is impacted, not just the low-frequency regions which is the 
most critical to the thermal conductivity at low temperatures. As a result, the specific heat also changes when impurities are present, but the magnitude of the changes does not correlate with the calculated changes in the thermal conductivity.

As expected, the largest thermal conductivity of Si occurs for defect-free ${ }^{28} \mathrm{Si}$ samples. The presence of impurities at the atomic percent level always reduces $\kappa$. We have considered a wide range of $\mathrm{Si}$ isotopes (most of which do not exist in nature but are convenient for the present calculations) and have shown that the drop in $\kappa$ is substantial for ${ }^{56} \mathrm{Si}$.

When actual impurities are introduced into the supercell, the drop in $\kappa$ is also isotope-dependent. We used 5.2 at.\% of substitutional $\mathrm{C}, \mathrm{Fe}$, and $\mathrm{Ge}$ in otherwise isotopically pure ${ }^{28} \mathrm{Si}$. The drop is relatively small in the case of substitutional $\mathrm{C}$ and Fe. Note that while Si samples can easily be doped with ${ }^{12} \mathrm{C}$ or ${ }^{13} \mathrm{C}$ impurities, it would be most challenging to grow a sample with the desired concentration of substitutional Fe. This impurity much prefers to remain interstitial.

The most interesting prediction deals with 5.2 at.\% of $\mathrm{Ge}$ in $\mathrm{Si}$, especially since the presence of substitutional $\mathrm{Ge}$ in $\mathrm{Si}$ does not affect very much the electrical conductivity (except for an increase in mobility). This impurity is almost always substitutional, samples of $\mathrm{Si}_{1-x} \mathrm{Ge}_{x}$ with a wide range of $x$ are common, although samples with the desired isotope combinations are not. Our prediction is that the thermal conductivities at $T=125 \mathrm{~K}$ of nanostructures of ${ }^{28} \mathrm{Si}_{0.95}{ }^{70} \mathrm{Ge}_{0.05}$ and ${ }^{28} \mathrm{Si}_{0.95}{ }^{74} \mathrm{Ge}_{0.05}$ should differ by a factor of $\sim 7$. Note that substantially increasing the Ge concentration may not affect the result very much (Fig. 8). Using nat $\mathrm{Ge}$ instead of isotopically pure ${ }^{70} \mathrm{Ge}$ or ${ }^{74} \mathrm{Ge}$ leads to $\kappa(T=$ $125 \mathrm{~K})=0.42 \mathrm{~W} / \mathrm{mK}$.

Although it is difficult to relate macroscopic quantities such as $\kappa$ to specific atomic-level processes, our results suggest that the key is the vibrational lifetime of some impurity-related localized vibrational modes. In particular, we find a strong correlation between the localization of the fully symmetric breathing mode of the four Si NN to the impurity and the relative change in the thermal conductivity in ${ }^{28} \mathrm{Si}_{182}{ }^{M} \mathrm{Si}_{10}$ in the range $10<M<110$. This suggests that some impurity isotopes are capable of trapping phonons.

\section{ACKNOWLEDGMENTS}

S.K.E.'s research is supported in part by the Grant No. D-1126 from the R.A. Welch Foundation. C.C.'s work is supported by Grant No. 03X0512D from the German Federal Ministry of Education and Research (BMBF). We thank Philip Howell and Matthias Scheffler for fruitful discussions. Texas Tech's High Performance Computer Center provided generous amounts of CPU time. *stefan.estreicher@ttu.edu

${ }^{1}$ H. J. Queisser and E. E. Haller, Science 281, 945 (1998).

${ }^{2}$ H. Temkin and S. K. Estreicher, in Encyclopedia of Chemical Physics and Physical Chemistry, edited by J. H. Moore and N. D. Spencer, Vol. III (IOP, Bristol, 2002), p. 2567.

${ }^{3}$ H. Huff, Encycl. Appl. Phys. 17, 437 (1996).

${ }^{4}$ T. M. Gibbons and S. K. Estreicher, Phys. Rev. Lett. 102, 255502 (2009); 103, 099904(E) (2009).

${ }^{5}$ M. Apostol, J. Appl. Phys. 104, 053704 (2008).

${ }^{6}$ Theory of Defects in Semiconductors, edited by D. A. Drabold and S. K. Estreicher (Springer, Berlin, 2007).

${ }^{7}$ W. J. Parker, R. J. Jenkins, C. P. Butler, and G. L. Abbot, J. Appl. Phys. 32, 1679 (1961).

${ }^{8}$ P. Carruthers, Rev. Mod. Phys. 33, 92 (1961).

${ }^{9}$ J. Callaway, Phys. Rev. 113, 1046 (1959).

${ }^{10}$ M. Budde, G. Lüpke, C. P. Cheney, N. H. Tolk, and L. C. Feldman, Phys. Rev. Lett. 85, 1452 (2000).

${ }^{11}$ G. Lüpke, N. H. Tolk, and L. C. Feldman, J. Appl. Phys. 93, 2317 (2003).

${ }^{12}$ K. K. Kohli, G. Davies, N. Q. Vinh, D. West, S. K. Estreicher, T. Gregorkiewicz, I. Izeddin, and K. M. Itoh, Phys. Rev. Lett. 96, 225503 (2006).

${ }^{13}$ D. West and S. K. Estreicher, Phys. Rev. Lett. 96, 115504 (2006).

${ }^{14}$ D. West and S. K. Estreicher, Phys. Rev. B 75, 075206 (2007).

${ }^{15}$ M. J. Gillan, Phys. Scr., T 39, 362 (1991).

${ }^{16}$ M. P. Allen and D. J. Tildesley, Computer Simulations of Liquids (Clarendon, Oxford, 2000).

${ }^{17}$ S. G. Volz and G. Chen, Phys. Rev. B 61, 2651 (2000).

${ }^{18}$ S. G. Volz, Phys. Rev. Lett. 87, 074301 (2001).

${ }^{19}$ S. G. Volz, J. B. Saulnier, G. Chen, and P. Beauchamp, Microelectron. J. 31, 815 (2000).
${ }^{20}$ S. G. Volz and G. Chen, Phys. Rev. B 61, 2651 (2000).

${ }^{21}$ H. Ishii, A. Murakawa, and K. Kakimoto, J. Appl. Phys. 95, 6200 (2004).

${ }^{22}$ J. Shiomi and S. Maruyama, Phys. Rev. B 73, 205420 (2006).

${ }^{23}$ S. Wang, X. Liang, X. Xu, and T. Ohara, J. Appl. Phys. 105, 014316 (2009).

${ }^{24}$ S. Volz, J. B. Saulnier, M. Lallemand, B. Perrin, P. Depondt, and M. Mareschal, Phys. Rev. B 54, 340 (1996).

${ }^{25}$ Y. Lee, S. Lee, and G. S. Hwang, Phys. Rev. B 83, 125202 (2011).

${ }^{26}$ S. Stackhouse, L. Stixrude, and B. B. Karki, Phys. Rev. Lett. 104, 208501 (2010).

${ }^{27}$ N. de Koker, Phys. Rev. Lett. 103, 125902 (2009).

${ }^{28}$ D. A. Broido, M. Malorny, G. Birner, N. Mingo, and D. A. Stewart, Appl. Phys. Lett. 91, 231922 (2007).

${ }^{29}$ A. Ward and D. A. Broido, Phys. Rev. B 81, 085205 (2010).

${ }^{30}$ J-W. Jiang, B-S. Wang, and J-S. Wang, Appl. Phys. Lett. 98, 113114 (2011).

${ }^{31}$ By. Kang and S. K. Estreicher (unpublished).

${ }^{32}$ D. Sánchez-Portal, P. Ordejón, E. Artacho, and J. M. Soler, Int. J. Quantum Chem. 65, 453 (1997).

${ }^{33}$ E. Artacho, D. Sánchez-Portal, P. Ordejón, A. García, and J. M. Soler, Phys. Status Solidi B 215, 809 (1999).

${ }^{34}$ C. Carbogno et al. (unpublished).

${ }^{35}$ V. Blum, R. Gehrke, F. Hanke, P. Havu, V. Havu, X. Ren, K. Reuter, and M. Scheffler, Comput. Phys. Commun. 180, 2175 (2009).

${ }^{36}$ N. Troullier and J. L. Martins, Phys. Rev. B 43, 1993 (1991).

${ }^{37}$ L. Kleinman and D. M. Bylander, Phys. Rev. Lett. 48, 1425 (1982).

${ }^{38}$ V. L. Moruzzi and C. B. Sommers, Calculated Electronic Properties of Ordered Alloys: A Handbook (World Scientific, Singapore, 1995). 
${ }^{39}$ D. M. Ceperley and B. J. Alder, Phys. Rev. Lett. 45, 566 (1980).

${ }^{40}$ J. P. Perdew and A. Zunger, Phys. Rev. B 23, 5048 (1981).

${ }^{41}$ J. P. Perdew, K. Burke, and M. Ernzerhof, Phys. Rev. Lett. 77, 3865 (1996).

${ }^{42}$ O. F. Sankey and D. J. Niklewski, Phys. Rev. B 40, 3979 (1989).

${ }^{43}$ O. F. Sankey, D. J. Niklewski, D. A. Drabold, and J. D. Dow, Phys. Rev. B 41, 12750 (1990).

${ }^{44}$ F. Widulle, T. Ruf, M. Konuma, I. Silier, M. Cardona, W. Kriegseis, and V. I. Ozhogin, Solid State Commun. 118, 1 (2002).

${ }^{45}$ M. Sanati and S. K. Estreicher, J. Phys. Condens. Matter 16, L327 (2004).

${ }^{46}$ M. Sanati, S. K. Estreicher, and M. Cardona, Solid State Commun. 131, 229 (2004).

${ }^{47}$ M. Cardona, R. K. Kremer, M. Sanati, S. K. Estreicher, and T. R. Anthony, Solid State Commun. 133, 465 (2005).

${ }^{48}$ R. K. Kremer, M. Cardona, E. Schmitt, J. Blumm, S. K. Estreicher, M. Sanati, M. Bockowski, I. Grzegory, T. Suski, and A. Jezowski, Phys. Rev. B 72, 075209 (2005).

${ }^{49}$ S. K. Estreicher, M. Sanati, D. West, and F. Ruymgaart, Phys. Rev. B 70, 125209 (2004).

${ }^{50}$ D. Li, Y. Wu, P. Kim, L. Shi, P. Yang, and A. Majumdar, Appl. Phys. Lett. 83, 2934 (2003).

${ }^{51}$ A. I. Hochbaum, R. Chen, R. D. Delgado, W. Liang, E. C. Garnett, M. Najarian, A. Majumdar, and P. Yang, Nature (London) 451, 163 (2008).
${ }^{52}$ R. Chen, A. I. Hochbaum, P. Murphy, J. Moore, P. Yang, and A. Majumdar, Phys. Rev. Lett. 101, 105501 (2008).

${ }^{53}$ A. I. Boukai, Y. Bunimovich, J. Tahir-Kheli, J.-K. Yu, W. A. Goddard III, and J. R. Heath, Nature (London) 451, 168 (2008).

${ }^{54}$ D. Donadio and G. Galli, Phys. Rev. Lett. 102, 195901 (2009).

${ }^{55}$ C. J. Glassbrenner and G. A. Slack, Phys. Rev. 134, A1058 (1964).

${ }^{56}$ G. Chen, Phys. Rev. Lett. 86, 2297 (2001).

${ }^{57} \mathrm{P}$. Howell (private communication).

${ }^{58}$ L. Wei, P. K. Kuo, R. L. Thomas, T. R. Anthony, and W. F. Banholzer, Phys. Rev. Lett. 70, 3764 (1993).

${ }^{59}$ M. Asen-Palmer, K. Bartkowski, E. Gmelin, M. Cardona, A. P. Zhernov, A. V. Inushkin, A. Taldenkov, V. I. Ozhogin, K. M. Itoh, and E. E. Haller, Phys. Rev. B 56, 9431 (1997).

${ }^{60}$ T. Ruf, R. W. Henn, M. Asen-Palmer, E. Gmelin, M. Cardona, H. J. Pohl, G. G. Devyatych, and P. G. Sennikov, Solid State Commun. 115, 243 (2000).

${ }^{61}$ J.-W. Jiang, J. Lan, J.-S. Wang, and B. Li, J. Appl. Phys. 107, 054314 (2010).

${ }^{62}$ M. Fèvre, A. Finel, R. Caudron, and R. Mévrel, Phys. Rev. B 72, 104118 (2005).

${ }^{63} \mathrm{~J}$. Weber (private communication).

${ }^{64} \mathrm{G}$. Davies (private communication). 